\title{
DIE ANGST ZUM LAUFEN BRINGEN - HERTA MÜLLERS TEXT-BILD-COLLAGEN UND IHRE POLNISCHEN ÜBERSETZUNGEN
}

\begin{abstract}
Making my fear run - Herta Müller's collage-poetry and its polish translations
Herta Müller, born in Rumania as a member of the German minority, began to create her collages after having immigrated to West Germany. Being heterogeneous combinations of words and pictorial elements cut out from print media, the collages can be considered as an attempt to forge a link between west German culture and life in Rumania under the dictatorship of Ceauşescu. An important role in the articulation of autobiographical experience plays a specific rhythm, which is characterized by intermodality and criss-crossed words and pictorial elements. Rhythm not only renders experience - as to speak subcutaneously - apt to be communicated, deliberate ruptures of rhythm indicate the traumatizing effects of past experiences and anxiety. The article analyses to what extent the polish translators Leszek Szaruga and Andrzej Kopacki take into account the structural complexity and intermediality of the collages.
\end{abstract}

KEYWORDS: Herta Müller, collages, intermediality, rhythm, translation

Spätestens seitdem sie 2009 den Nobelpreis für Literatur erhielt, ist die rumäniendeutsche Autorin Herta Müller einer breiten Öffentlichkeit bekannt, vor allem durch ihre prosaischen Werke und die literaturkritischen und politischen Essays. Weniger bekannt sind ihre lyrisch-künstlerischen Arbeiten, die Collagen. ${ }^{1} 1993$ wurde in Form einer Postkartensammlung der erste Einzelband Der Wächter nimmt seinen Kamm veröffentlicht ${ }^{2}$, auf den 2000 der Band Im Haarknoten wohnt eine Dame folgte. ${ }^{3} 2005$ und 2012 erschienen die beiden bisher letzten Collagen-Bände: Die

\footnotetext{
${ }^{1}$ Erstmals veröffentlichte Müller in ihrer Poetik-Vorlesung einige ihrer Text-Bild-Gebilde, pflegte aber schon vorher einzelne Essaybände oder den Beginn von Buchkapiteln mit Collagen zu illustrieren. U.a. in: Herta Müller: Der Teufel sitzt im Spiegel. Wie Wahrnehmung sich erfindet, Berlin 1991.

${ }^{2}$ Herta Müller: Der Wächter nimmt seinen Kamm, Reinbek bei Hamburg 1993.

${ }^{3}$ Herta Müller: Im Haarknoten wohnt eine Dame, Reinbek bei Hamburg 2000.
} 
blassen Herren mit den Mokkatassen und Vater telefoniert mit den Fliegen. ${ }^{4}$ Diese liegen inzwischen auch in der polnischen Übersetzung von Leszek Szaruga vor, einige Collagen wurden überdies von Andrzej Kopacki ins Polnische übertragen. ${ }^{5}$

Herta Müllers Collagen-Technik entwickelte sich kontinuierlich weiter: während in den frühen Collagen der Text dominiert, stehen die Bildteile in den letzten Bänden nahezu gleichberechtigt neben den Textelementen. Zudem ist die Grenze zwischen Bild und Text nicht mehr offensichtlich, die Bildausschnitte ragen in die Texte und sind mit ihnen verzahnt. Diese visuelle Rahmenlosigkeit als Entgrenzung des Bildes in den Text hinein bewirkt das Entstehen hybrider Gebilde. Auch durch die Anreicherung der Schrift mit visuellen Elementen, das visuelle In-Szene-Setzen der Schrift wird die mediale Überschreitung aktiv gehalten. Die einzelnen, durch Größe, Farbigkeit und typografische Ausgestaltung betonten Schriftelemente gehen ein Wechselspiel mit den Bildelementen ein. Das mediale Nebeneinander wird somit durch die Koppelung von Text und Bild überschritten und die Dominanz des Textes durch eine Überführung der Medienleistung von Bild und Text ineinander gebrochen, womit die Collagen für eine intermediale Analyse interessanter werden. Die heterogene, zwischen Bild und Text oszillierende Struktur der Collagen hintertreibt eine eindeutige Rezeptionshaltung, denn die hybriden Gebilde lassen sich weder im Modus der Bilderschau noch des Lesens aufnehmen. Die Autorin lässt offen, ob ihre Collagen als Bilder oder Texte rezipiert werden sollen und betreibt bereits in der medialen Darbietung ein hintergründiges Spiel mit den medialen Kontexten. Während die ersten Collagen als einzeln herausnehmbare Postkarten in einer Box dargeboten wurden, werden die späteren in Buchform präsentiert, womit eine Verschiebung vom Rezeptionsmodus der bildenden Kunst hin zu einem literarischen Kontext suggeriert wird. Die mediale Hybridität von Müllers Text-Bild-Collagen wird auch dadurch akzentuiert, dass sie sowohl als Kunstwerke in Ausstellungen präsentiert als auch in Lesungen als Texte zu Gehör gebracht werden. Die mediale Schwelle zwischen Text und Bild, Sprach- und Bildkunstwerk wird so ständig umspielt, wobei sich jedes Medium gegenüber dem anderen definiert und positioniert.

Die bisher kurz skizzierten, in Müllers Collagen inszenierten medialen Grenzüberschreitungen sollen im Fokus meiner Ausführungen stehen. Die in den Collagen inszenierte, vibrierende Gleichzeitigkeit von Bild und Text soll dabei auf psychologische Dispositionen der Autorin rückbezogen werden, die in der von Angst geprägten Atmosphäre der rumänischen Diktatur gründen. In ihren Interviews spricht die im Rumänien Ceauşescus zermürbenden Verfolgungen ausgesetzte Autorin von der lähmenden Angst, die den Zeitverlauf zu Schreckensbildern gefrieren lässt, und die

\footnotetext{
${ }^{4}$ Herta Müller: Die blassen Herren mit den Mokkatassen, München 2005; Herta Müller: Vater telefoniert mit den Fliegen, Frankfurt a.M. 2012.

${ }^{5}$ Herta Müller: Kolaże, aus dem Deutschen übers. von Leszek Szaruga, Wrocław 2013; Herta Müller: Kolaże, aus dem Deutschen übers. von Andrzej Kopacki, in: Literatura na Świecie 2014, nr 1-2, S. 133-156.
} 
zugleich die Initialzündung des Schreibens darstellt, will dieses doch gegen die „stehengebliebene Zeit ${ }^{\text {“6 }}$ der Diktatur angehen:

Ich glaube, die Angst, die still hält, ist am gefährlichsten. [...] Ich habe mich immer darum bemüht, dass die Angst sich bewegt. Und das Schreiben habe ich damals angefangen, um mir selber zu versichern, daß die Angst laufen lernt. ${ }^{7}$

Dieses Widerspiel einer Erstarrung in Schreckensbildern und dem Wunsch, sich von diesen freizuschreiben, wird in den Collagen mit ihrem Oszillieren zwischen Bild und Text, Stillstand und Bewegung inszeniert. Die stillgelegte Bildlichkeit der Collagen ist als Korrelat der erstarrten Zeit lesbar, der Schrecksekunde, die nie vorrübergeht, wie der Stillstand der Zeit beim Verhör durch den Geheimdienst Securitate. Zwischen Erstarrung und Schreibbewegung sollen Müllers Text-Bild-Hybriden im Folgenden verortet werden.

\section{Phantasmagorien des Schreckens}

Das Schreiben - so Müller - vollzieht sich in Bildern. ${ }^{8}$ Es gibt in Müllers Prosa genau genommen keinen Fortschritt, keine Entwicklung, sie bleibt vielmehr den immer gleichen, obsessiv wiederkehrenden Bildern verhaftet, in denen das eigene Erleben sich kristallisiert. Auch in ihre Collagen komponiert Müller immer wieder Männer in Trenchcoats und ins Gesicht gezogenen Hüten, die als Schattenrisse und vage Silhouetten gesichtslos durch die Bild- und Textelemente geistern, ja recht eigentlich zu ihren Hauptakteuren werden. Erzählprosa, Essays und Collagen schöpfen aus dem gleichen Arsenal von Bildern und Vorstellungen, sind über traumatisch verdichtete Wörter und Bildvorstellungen wie dem „Koffer“ oder dem KönigDiktator miteinander vernetzt. Die Schreckensbilder der Vergangenheit irrlichtern durch Wort- und Bildelemente der Collagen und verflechten sie zu einer Phantasmagorie des Schreckens. Das intermediale Verfahren generiert ein freies Flottieren der Signifikanten, in dem die einzelnen Gebilde lediglich kontingente Arretierungen darstellen. In den Collagen kommt damit eine Ästhetik der Brüche und subkutan wirkender Vorstellungs- und Bildkomplexe zum Tragen, die über die medialen Grenzen hinweg die Bild- und Wortelemente miteinander verwirken.

\footnotetext{
${ }^{6}$ Vgl. Ich habe die Sprache gegessen. Die Literatur-Nobelpreisträgerin Herta Müller über ihren zusammengeklebten Gedichte und über die Macht und das Versagen der Wörter, in: Der Spiegel 2012, 35, S. 128-132, hier S. 131.

${ }^{7}$ Herta Müller, Beverly Driver Eddy: Die Schule der Angst. Gespräch mit Herta Müller vom 14. April 1998, in: The German Quaterly 1999, 72.4, S. 329-339, hier S. 332.

${ }^{8}$ Vgl. Müller: Der Teufel sitzt im Spiegel, S. 83.
} 
Ihre Schreckensbilder bezieht Müller aus den bundesrepublikanischen Printmedien. Durch das Zerschneiden kann das transgressive Vermögen der Worte und Bilder entfaltet und Wahrnehmung neu in Bewegung versetzt, „erfunden“ werden. Durch das kombinatorische Verfahren stoßen die Wörter und Bilder der ausgeweideten Zeitschriften auf die Schreckensbilder im eigenen Kopf, die als unvermutet und blitzartig hervortretende Flash-Blacks die Collagen besiedeln. Der mediale ,Trash wird so ,zugeschnitten', dass sich jenseits der Augenfälligkeit und Gefälligkeit des äußerlich Aufgeprägten, in den Brüchen und porösen Stellen, der Schmerz einnisten kann, von dem Müllers Schreiben seinen Anfang nimmt und aus dem es seine Legitimation bezieht. Das vorgefundene Sprach- und Bildmaterial wird so für die Verwundungen durchlässig, die die Realität - die der Diktatur und die westdeutsche den Menschen antut. So finden sich die Wort-und Bildelemente unter Müllers Schere beharrlich zu bedrückenden Schreckensvisionen zusammen, in denen die Angst in den ästhetischen Modus der Repräsentation hinübergerettet wird und zugleich eine ästhetische Verdichtung erfährt. Die verzerrten Größenverhältnisse und Isolierung von Details, die sich nicht zu stabilen Entitäten fügen, sondern zu bizarren Ensembles addieren, verleihen den Collagen ein surrealistisches Gepräge. Der mehrfach konstatierte, spezifische Surrealismus von Müllers Text-Bild-Collagen ${ }^{10}$ speist sich jedoch nicht aus den Ideologemen der Avantgarde, sondern ist als Korrelat des Grunderlebnisses aus der Diktatur zu begreifen: „Die Welt baute sich Stück für Stück zusammen gegen den Verstand. “11 In einem Interview erklärt Müller, Angst bewirke, dass die Wirklichkeit surreal wird: „Angst macht große Augen, die Dinge werden fremd.“12 Der unter der Diktatur erworbene ,fremde Blick“ rührt vom zermürbenden Terror des Überwachungsstaates, dem Ferment, in dem die Deformierungen der Menschen, der „Verlust von Selbstverständlichkeit und Angst ${ }^{\text {“13 }}$ gedeihen können. Die Angst ist es, die den Schleier der Wirklichkeit zerreißt und ein Gefühl schillernder Irrealität erzeugt, der Welt ihre Natürlichkeit nimmt und sie in heterogene Bruchstücke zerbröckeln lässt: „Die Einheit der Dinge mit sich selbst hatte ein Verfallsdatum. Alles rundum schien sich nicht mehr sicher zu sein, ob es das, oder dies oder etwas ganz anderes war. ${ }^{\text {" } 14}$ Durch das Flickwerk der Collagen scheint das Phantasma einer alptraumhaft unverständlichen, sinnlosen und absurden Welt.

\footnotetext{
${ }^{9}$ Ebd., S. 19.

${ }^{10}$ Die Verwandtschaft mit dem Surrealismus ist Müller mehrfach bescheinigt worden (vgl. Jürgen Wertheimer: Im Papierhaus wohnt die Stellungnahme. Zu Herta Müllers Bild-Text-Collagen, in: Herta Müller. Text + Kritik 155, hrsg. von Heinz-Ludwig Arnold, München 2002, S. 80-84, hier S. 81).

${ }^{11}$ Herta Müller: Der fremde Blick oder Das Leben ist ein Furz in der Laterne, Göttingen 1999, S. 5.

${ }^{12}$ Müller: Ich habe die Sprache gegessen, S. 131.

${ }^{13}$ Herta Müller: Mit dem Auge kann man keinen Stift halten, <http://derstandard.at/1537469>, [abgerufen am 03.07.2014], S. 1-3, hier S. 1.

${ }^{14}$ Müller: Der fremde Blick, S. 5.
} 


\section{Laufen lernen - Kontingente Spiele}

Um das Leben in der surrealen Welt der Angst ertragen zu können, brauche sie die Konvention des Spiels, erklärt Müller in einem Interview mit dem polnischen Übersetzer Kopacki. ${ }^{15}$ Der Hinweis auf die Konvention des Spiels scheint die Affinität zu den Sprachexperimenten und Collagen der literarischen Avantgarde der 20er Jahre, beispielsweise des Dada zu belegen. Die Verbindung scheint berechtigt, macht sich Müller doch den befreienden Impetus der Collage avantgardistischer Provenienz zu eigen. ${ }^{16}$ Auf das spielerische Moment der Collagen, den Humor, den sie in ihrer Prosa nicht zum Ausdruck bringen könne, verweist die Autorin selbst. ${ }^{17}$ Das befreiende Lachen steht jedoch immer an der Schnitt-Stelle von Humor und Angst, im Hintergrund der spielerischen Kombinatorik der Collagen lauert der tiefe Ernst, so dass sich Angst und Spiel letztendlich die Waage halten.

In den Collagen spielt Müller solche kombinatorischen Spiele, die sie von der Angst befreien sollen. Die Konnotationen zum Spiel sind vielfältig. Zum Beispiel ist der Tisch, der als Schauplatz der Wortspielereien mehrfach als Bildelement erscheint, kariert wie ein Schachbrett, und auch im Textteil erscheinen schwarzweiß karierte Tische, auf denen die Figuren hin- und hergeschoben werden. Wenn die Wörter auf dem Tisch verrückt werden wie beim Schachspiel und dabei dem Zufall die Regie überlassen wird, ist dies zunächst ein Bild für das Eingestehen der Kontingenz, der „Zufallsblindheit“ der Sprache, um einen Ausdruck Rorty's zu gebrauchen. ${ }^{18}$ Indem sie den Wörtern folgt, „dahin, wo sie hinführen“, und sie in immer neuen Konfigurationen zusammenfügt, gelingt es Müller, die zufallsblinde Prägung der Sprache gleichsam zu ertasten. Der Zufall ist hier doppelt besetzt: zum einen als Angst vor der Kontingenz der Sprache, hinter der das Phantasma der Sinnlosigkeit lauert: „Da sieht man noch mehr, als wenn man aufs Papier schreibt, wie sehr alles dem Zufall gehorcht. [...] Man könnte Angst kriegen, wenn man sich darüber Rechenschaft gibt, wie viel an diesem Zufall liegt." "19 Auf der anderen Seite liegt in dieser Lotterie mit Worten ein befreiendes Moment, wird hier doch eine „EntGötterung" (Rorty) der Sprache im Sinne eines Herauslösens aus ideologischen Prägungen vollzogen. In den Collagen spielt Müller ihre eigenen Sprachspiele, die sie denjenigen der Diktatur und der westdeutschen Medien entgegensetzt. Im Zu-

\footnotetext{
${ }^{15}$ Szczegót nie kłamie, z Herta Müller rozmawia Andrzej Kopacki, in: Literatura na Świecie 2014, nr 1-2, S. 157-163, hier S. 160.

${ }^{16}$ Vgl. Wolfgang Max Faust: Bilder werden Worte. Zum Verhältnis von bildender Kunst und Literatur vom Kubismus bis zur Gegenwart, Köln 1987, S. 53f.

${ }^{17}$ Vgl. Müller: Ich habe die Sprache gegessen, S. 130.

${ }^{18}$ Richard Rorty: Kontingenz, Ironie und Solidarität, aus dem Amerikanischen von Christa Krüger, Frankfurt a.M. 1989, S. 61

${ }^{19}$ Müller, Eddy: Die Schule der Angst, S. 338.
} 
sammensuchen neuer Wörter werden die Collagen zum nie endenden Versuch, der Sprache eine eigene, unverwechselbare Prägung aufzudrücken. ${ }^{20}$

Indem sie sich der „schiere(n) Kontingenz“ der Sprache und Bilder überlässt, entfesselt Müller die poetische Fliehkraft des Wort- und Bildmaterials, die sich nicht ideologisch in den Dienst nehmen lässt. Müllers Sprachspiele sind aus dem Zufall geboren - die Autorin lässt sogar das Konzept der 'écriture automatique' dadaistischer Provenienz anklingen ${ }^{21}$, wenn sie die Regie ganz den Wörtern auf ihrem Tisch überlässt: „Weil die Wörter schon vorhanden sind, denke ich manchmal, dass ich es gar nicht bin, die schreibt. ${ }^{\text {‘22 }}$ Gerade die Unberechenbarkeit der Elemente, mit der durch den unverhofften Zusammenprall der Elemente die poetische Kraft freigesetzt wird, stellt ihren Vorzug dar, denn die auf diese Weise befreiten Wörter werden nicht nur aus alten, medial verordneten Zusammenhängen herausgeschleudert, sondern können in neuen Sinnzusammenhängen zueinander finden: „wenn ich diese gedruckten Wörter ausgeschnitten auf dem Tisch liegen habe, fliegen sie zusammen und werden eine Geschichte ${ }^{\text {“23 }}$ Müller puzzelt sich also ihre Sprachspiele in der Hoffnung zusammen, dass durch diese zufallsgeschuldeten Arrangements ein poetischer Mehrwert entsteht. Die Collagen werden damit zum Schauplatz von ,ästhetischen Fluchtbewegungen“'24, bilden die 'Schnitt-Menge' ideologisch vorgeprägter Wirklichkeitswahrnehmung und der poetischen Eigenbewegung der Sprache. Damit es nicht zum medial und ideologisch zugerichteten Stillleben erstarrt, wird das Geschriebene stets aufs Neue durchgeschüttelt und durcheinandergewirbelt. Dieses Verfahren der fortwährenden Neuanordnung, in der Realitätspartikel frei durch die Text- und Bilderwelt der Collagen vagabundieren und zu neuen Sinnzusammenhängen vernetzt werden, korrespondiert mit dem poetologischen Konzept der „erfundenen Wahrnehmung", die sich als ein Unterwegssein entfaltet, und sich in die Lage versetzt, die Verhältnisse der Realität immer wieder neu zu entwerfen.

In den späteren Collagen wird die Textbewegung durch Reime und Metrum in Bewegung gehalten. Der Reime wird bezeichnenderweise von der Autorin als das eigentliche Movens der Textbewegung angesehen: „Er (der Reim, B.S.) ist wie ein kleiner Motor. Er schiebt das Ganze an. ${ }^{، 25}$ Es scheint also vor allem die prosodische, rhythmisierende Funktion des Reims wichtig zu sein, die mit dem pulsierenden Takt

\footnotetext{
20 "Dann wird man der Sprache keine eigene Prägung gegeben, sondern ein Leben lang nur vorgeprägte Stücke herumgeschoben haben.“ (Rorty: Kontingenz, Ironie und Solidarität, S. 53).

${ }^{21}$ Vgl. Tristan Tzara: Dada Manifest über die aschwache Liebe und die bittere Liebe, in: ders.: 7 DADA Manifeste, Hamburg 1978, S. 44.

${ }^{22}$ Müller: Ich habe die Sprache gegessen, S. 130.

${ }^{23}$ Müller, Eddy: Die Schule der Angst, S. 338.

${ }^{24}$ Philipp Müller: Fluchtlinien der erfundenen Wahrnehmung. Strategien der Überwachung und minoritäre Schreibformen in Herta Müllers Roman „Heute wäre ich mir lieber nicht begegnet“, in: Herta Müller. Text + Kritik 155, hrsg. von Heinz-Ludwig Arnold, München 2002, S. 49-58, hier S. 51.

${ }^{25}$ Müller: Ich habe die Sprache gegessen, S. 130.
} 
des Metrums zusammenwirken und die Dynamik der Texte aktiv halten soll. Der Reim hält die Textbewegung als akustisch wirksames Muster in Gang. ${ }^{26}$ Die Collagen sind von einer Lauttextur aus akustisch wahrnehmbaren Elemente wie Binnenreimen, Alliterationen, Anaphern und Lautmalerei durchzogen, wobei der Reim ein zentrales Organisationsmuster bildet: Je mehr die konventionelle Grammatik und Semantik an Bedeutung für die Textorganisation verlieren, desto wichtiger wird das Strukturierungsprinzip des Reims, der als formal organisierendes Prinzip gleichsam ersatzweise die Rolle syntaktischer Regeln und semantischer Bezüge übernimmt. Zwar verklammert der Reim die zerschnittenen Sätze, fügt sie allerdings nicht zu soliden Sinnverbunden zusammen. Ebenso wie metrische Muster nur den trügerischen Schein einer Ordnung vermitteln, halten die Reime die Texte nur scheinbar zusammen und werden nicht Kohärenz stiftend wirksam. Das genaue Gegenteil ist der Fall: Indem der Reim ein spielerisches Vernetzen sinnfremder Wörter erlaubt, ermöglicht er den Einbruch der Kontingenz in eine begrifflich sanktionierte Weltsicht und induziert so die Befreiung der Sprache von überkommenen Zusammenhängen. Das hier inszenierte Zufallsspiel der Reime bringt zum einen das Sprachmaterial in Bewegung und aktiviert die poetische Eigenkraft der Sprache, birgt aber auch das Risiko des Sinnverlusts, indem es die Kontingenz der Sprache sinnfällig macht und den Eindruck der „Zufallsblindheit“ der sprachlichen Ordnungen unterstützt. Auch die Verteilung der Farben über die Collagen erweist sich als arbiträre Streuung, die einer etwaigen Semantik der Farben zuwiderläuft. Wie Reimmuster und Versmaß suggerieren die Farbmuster Bedeutung nur, letztendlich sind Farben genauso wie die Wörter kontingent, und werfen ebenso wie Typografie und Faktur ein brüchiges Netz über die Collagen. Die übereinander geblendeten Strukturierungen sind häufig gegenläufig und lassen sich nicht zu stabilen Sinnzusammenhängen vernetzen. So kollidiert beispielsweise die Akzentuierung einzelner Elemente durch den Reim mit den visuellen Hervorhebungen. Paradoxe Verbindungen, wenn die Inszenierung der Schrift sich konträr zur Semantik verhält, indem etwa das Wort „klein“ in überdimensionalen Lettern erscheint, entfalten eine sinndestruierende Wirkung. Mit den Inszenierungen der Sprachsegmente wird überdies unaufhörlich auf ihre mediale Verfasstheit verwiesen. Durch das Proliferieren der typografischen Hervorhebungen, in dem sich nahezu jedes Wort dem Betrachter gleichsam aufdrängt, wird die Schlagzeilenoptik der Printmedien zum Implodieren gebracht und eine Zertrümmerung von Sinnbezügen induziert. Es wird eine irritierende Mischung aus visuell erzeugter Eindringlichkeit und semantischer Vieldeutigkeit zustande gebracht.

Wenn über ähnliche Farben oder Typografie Bezüge hergestellt werden, treten die linearen Strukturierungen der Textsegmente zugunsten der visuellen zurück, die

\footnotetext{
${ }^{26}$ Schreiben ist ja für Müller immer auch Hören, sie bekennt, alle ihre Texte laut zu lesen, oder sie im Kopf zu „hören“ (vgl. ebd., S. 130).
} 
sich zu einer komplexen Textur vernetzen. Diese konstellative Struktur der Texte, die neben der horizontalen auch vertikale Lesarten provoziert, führt zu einer Überlagerung von potenziellen Verknüpfungen, die syntaktische Mehrbezüglichkeiten hervorbringt. Indem die Texte von der linearen Lesbarkeit entbunden werden, werden die medialen Beschränkungen des Textes aufgehoben. Durch das Überführen der Texte aus der Linearität in ganzheitlich wahrnehmbare Konstellationen wird der Eindruck der Gleichzeitigkeit forciert und eine simultane Wahrnehmung wie bei der Bildbetrachtung herausgefordert. Einzelne Wörter in greller Farbe oder überdimensionaler Größe dominieren die Texte, springen den Leser/Betrachter förmlich an. Weniger typografisch hervorgehobene Elemente treten nicht nur optisch in den Hintergrund, sondern beanspruchen die Aufmerksamkeit des Betrachters mit zeitlicher Verzögerung. Es wird somit nicht eigentlich Simultanität erzeugt, sondern eine alternative Sukzessivität, die mit der linearen Textbewegung kollidiert. Die heterogene, zwischen Bild und Text oszillierende Struktur der Collagen hintertreibt eine eindeutige Rezeptionshaltung: Der Rezeptionsmodus schwenkt beständig in einer Art Kippbewegung vom Modus des Betrachtens zur Lesebewegung und verharrt in einer vibrierenden Gleichzeitigkeit von Sukzessivität und Simultaneität. Auch die Relationen der Wort- und Bildelemente sind von irisierender Vieldeutigkeit. Konventionelle Erwartungshaltungen, nach denen Text und Bild sich als Erklärung, Doppelung oder zumindest kontrastive Spannung auseinander entfalten, werden enttäuscht. Wenn das Muster der Illustrierten aufgegriffen wird, dann nur, um es zu zersetzen. Trotz entfernter illustrativer Verweise bleiben die Relationen zwischen Text und Bild vage und rätselhaft. Da statt erhellender Kommentare neue Rätsel aufgegeben werden, wird der Leser immer wieder auf seinen Status als Betrachter zurückverwiesen. Es bleibt ihm überlassen, zum Leser zu werden und Rat beim Text zu suchen, wechselseitige Referenzen zwischen Bild und Text zu entwerfen - oder es beim reinen Betrachten zu belassen. Durch die Bezüge zwischen Text und Bild wird - gerade weil sie so vieldeutig sind - der Transfer zwischen den Medien permanent aktiv gehalten und eine intermediale Synthese unterlaufen. Das wechselseitige Durchdringen als ununterbrochener Prozess des Transformierens von Texten in Bilder und umgekehrt erzeugt vielmehr eine mediale Hybridität, die nahezu unendliche Verweise generiert.

Durch die kontrafaktorischen textuellen und visuellen Strukturierungen, die durch die redundante Typografie, Faktur und Farbe gebildet werden, wird die durch Metrum und Reim dynamisierte Textbewegung immer wieder abgebremst und erstarrt zu visuellen Arrangements, in denen die einzelnen „polysemen BruchStücke، ${ }^{627}$ in der Relation der Fremdheit zueinander stehen und sich zu absurden und erschreckenden Tableaus vernetzen. Der durch den Reim erzielte kindliche Ton und

\footnotetext{
${ }^{27}$ Norbert Otto Eke: Schönheit der Verwund(er)ung. Herta Müllers Weg zum Gedicht, in: Herta Müller. Text + Kritik, hrsg. von Heinz-Ludwig Arnoldt, München 2002, S. 64-79, hier S. 74.
} 
die tänzerische Selbstvergessenheit einer sich aus fixen Sinnbezügen herausschreibenden Sprachbewegung sind damit nur eine notdürftige Verhüllung des Schreckens einer unbegreiflichen Welt. Dieser bildet das eigentlich Authentische, das immer wieder ,aus dem Gemachten heraus(springt). ${ }^{\text {‘28 }}$ Wie in den Gedichten der bewunderten Lyrikerin Inge Müller wird so in Müllers gereimten und geleimten Gebilden der Eindruck einer ,,hüpfenden Zerbrochenheit ${ }^{\text {‘29 }}$ zustande gebracht, der biografisch verankert ist: Noch in ihrem neuesten Text Mein Vaterland war ein Apfelkern beschreibt Müller die Strategie der Angstbewältigung durch sinnlose Reime. ${ }^{30}$ Der spielerische Duktus der Collagen darf also nicht darüber hinwegtäuschen, dass Müller ihre Wörter um der Sprachmächtigkeit der Opfer willen zusammensucht. So erzählen die „Geschichten“, zu denen die Wörter und Bilder „zusammenfliegen“, von Beschädigungen und die grazile Leichtigkeit des Nonsens hält sich die Waage mit bedrückenden Vorstellungsbildern, zu denen sich die dergestalt befreiten Wörter mit unbeirrbarer Beharrlichkeit verdichten, wobei sie dann freilich alles Zufällige abstreifen.

Das kontrafaktorische Widerspiel von textuell-akustisch wirksamen Elementen und den auf visueller Ebene erkennbaren Mustern lässt sich mit der intermodalen Kategorie des Rhythmus fassen, wie sie von Michael Lommel in den Blick genommen wird. ${ }^{31}$ Auf die synästhetische Dimension des Rhythmus ist bereits verwiesen worden. ${ }^{32}$ Der Rhythmus kann mehrere Sinne durchqueren, ist sowohl visuell als auch akustisch wahrnehmbar. Als ,sinnesübergreifender sensus communis, der sich nicht allein durch Sukzession, durch ein Nacheinander, sondern ebenso durch vertikalen Austausch der Sinnesvermögen artikuliert، ${ }^{\text {33 }}$, ist der Rhythmus als Beschreibungskategorie der hochgradigen Komplexität intermedialer Gebilde angemessen. Rhythmik kommt in Müllers Collagen als komplexes Organisationsprinzip zum Tragen, der Rhythmus vereinigt die prosodisch-textuellen und die bildlich-visuellen Strukturierungen und trägt auch ihren Wechselwirkungen Rechnung. Durch lautliche und visuelle Rhythmisierung werden die Collagen in eine Struktur gebracht und davor bewahrt, in amorphe Gebilden zu zerfallen. In seiner intermodalen Wirksam-

\footnotetext{
${ }^{28}$ Herta Müller: Hunger und Seide, Reinbek bei Hamburg 1995, S. $36 \mathrm{f}$.

${ }^{29}$ Vgl. Herta Müller: In der Falle, Göttingen 1996, S. 46.

${ }^{30}$ Herta Müller: Mein Vaterland war ein Apfelkern. Ein Gespräch mit Angelika Klammer, München 2014, S. 8.

${ }^{31}$ Vgl. Michael Lommel: Der Rhythmus als intermodale Kategorie, in: Intermedialität analog/digital. Theorien - Methoden - Analysen, hrsg. von Joachim Paech und Jens Schröter, München 2008, S. 79-90.

${ }^{32}$ Vgl. Gilles Deleuze: Francis Bacon - Logik der Sensation, aus dem Franz. übers. von Joseph Vogl, München 1995, S. 31; Maurice Merleau-Ponty: Phénoménologie de la Perception, Paris 1966, S. 270; vgl. Bernhard Waldenfels: Sinnesschwellen: Studien zur Phänomenologie des Fremden, Frankfurt a.M. 1999, S. $73 \mathrm{f}$.

${ }^{33}$ Lommel: Der Rhythmus als intermodale Kategorie, S. 83.
} 
keit trägt der Rhythmus dem synästhetischen, auf visueller, lautlicher und taktiler Ebene operierenden Verfahren Müllers Rechnung, wenn wir Synästhesie als ein stetes Wechselspiel zwischen den Sinnen begreifen, einen komplexen Vorgang, der eine „maximale Beteiligung aller Sinne mit einschließt. ${ }^{\text {‘34 }}$ Müller betont den ,sinnlichen" Charakter der Arbeit mit den ausgeschnittenen Wörtern, die in ihrer Materialität wahrgenommen, die Hand genommen werden, beschreibt die Glätte des Papiers, seine Faktur, hebt also neben dem visuellen und akustischen das taktile Moment hervor. Müllers synästhetisches Verfahren steht allerdings in schroffem Gegensatz zu Vorstellungen von einer ursprünglichen Einheit der Sinne. Zwar kommen in den Collagen synästhetische Verfahren zum Tragen, allerdings werden Brüche und Spannungen zwischen den Sinnesmodalitäten einbezogen: Sehen und Hören kollidieren miteinander. Es scheint also, dass mit Blick auf intermediale Gebilde wie Müllers Collagen das Konzept der Synästhesie neu überdacht werden muss. Ein mediensynästhetischer Blick ${ }^{35}$ auf die Text-Bild-Collagen fördert gerade nicht Korrespondenzen im Sinne eines harmonischen Zusammenklangs zu Tage, sondern Hybridisierungen als Überschneidungen von Sinnesdispositiven, werden doch von Müller Passagen, Zwischenräume und Risse zwischen den einzelnen Sinnen exponiert und anthropologisiert - ganz im Sinne des Diktums: „Unsere Augen liegen so, dass beim Schauen der Riss im Bild drin ist. ${ }^{* 36}$ Der Rhythmus als intermodale Kategorie umfasst nicht nur das Zusammenspiel der Sinne, sondern auch die Brüche und Risse zwischen den Sinnesmodalitäten. Damit wird mit der Beschreibungskategorie des Rhythmus auch das kontrafaktorische Widerspiel von Reimen als akustisch wirksamen Elementen und den auf visueller Ebene erkennbaren Mustern fassbar, das Müllers Collagen auszeichnet. Indem sie die übereinander geblendeten bildlich-visuellen und textuell-lautlichen Strukturierungen integriert, erlaubt es die intermodale Kategorie des Rhythmus, die Collagen in ihrer medialen Grenzüberschreitungen zu erfassen. Auf die intermodale und medienüberschreitende Dimension des Rhythmus verweist Monika Schmitz-Emans. ${ }^{37}$ Eine „Poetik des Rhythmus“, die die rhythmische Organisierung der visuellen und akustischen Sphäre umfasst ${ }^{38}$,

\footnotetext{
${ }^{34}$ Lydia Andrea Hartl: Sprache, Rhythmus, Schrift. Medienkämpfe um Aug' und Ohr, in: Bernhard Banoun u.a. (Hg.): Aug' um Ohr. Medienkämpfe der österreichischen Literatur des 20. Jahrhunderts, Berlin 2002, S. 9-42, hier S. 16.

${ }^{35}$ Vgl. Michael Lommel: Stimme und Blick. Paradoxien synästhetischer Medienrezeption, in: Navigationen 2002, Jg. 1, Nr. 2, S. 7-16; Michael Lommel: Samuel Beckett-Synästhesie als Medienspiel, München 2006, S. 13-22.

${ }^{36}$ Müller: Der Teufel sitzt im Spiegel, S. 76.

${ }^{37}$ Monika Schmitz-Emans: Rhythmisierung als Musikalisierung. Zu Selbstbeschreibungen und ästhetischer Praxis in der experimentellen Dichtung des 20. Jahrhunderts, in: Colloquium Helveticum 2002, 32, S. 243-887.

${ }^{38}$ Vgl. ebd., S. 247, 257.
} 
macht sie u.a. in den Manifesten des Futurismus, des Dada und der konkreten Poesie aus. Schmitz-Emans hebt die Arbeit an der Entgrenzung, Entdifferenzierung der Künste und der Engführung der künstlerischen Ausdrucksmittel hervor, die durch rhythmisch-strukturierende Gestaltungspraktiken geleistet wird. ${ }^{39}$ Wie die Lyriker der Avantgardebewegungen sucht Herta Müller durch die ostentative rhythmische Strukturierung ihrer Collagen Anschlussstellen zwischen den Künsten und erprobt die Kombinierbarkeit ihrer materiell-medialen Grundlagen. Ihre Collagen setzen ein polyrhythmisches Geschehen in Gang, „,bei welchem der Rhythmus die Ehe zwischen Sprache und Bild stiften soll.“40 Müller ist es jedoch nicht darum zu tun, die Medien Bild und Sprache nahtlos ineinander aufgehen zu lassen. Vielmehr werden auch hier Brüche und Spannungen exponiert und ein Oszillieren zwischen Bild und Text, Stillstand und Bewegung erzeugt.

Für den Rhythmus sind ja Bewegung und Wiederholung konstitutiv, er ist Körperbewegung in Raum und Zeit. ${ }^{41}$ Die ,durch Wiederholung und Wiederholungserwartung geordnete" rhythmische Gestalt ist eine dynamische Einheit ${ }^{42}$ und keine statische Anordnung. Indem das Medium Text zum Trägermedium eines Rhythmus gemacht wird, wird es dynamisiert und vor der Erstarrung bewahrt. Im Gegensatz zum Pulsieren des Metrums, das voraussehbar ist, stört der Rhythmus jedoch die selbstgeschaffenen Ordnungen, nimmt auch Gegenbewegungen in sich auf und integriert Brüche und Kanten. ${ }^{43}$ Gerade in der Kunst des 20. Jahrhunderts lässt der Rhythmus das Einbrechen regelwidriger Strukturen zu - so treten beispielsweise in den freien Rhythmen moderner Lyrik Störungen, Gegenrhythmen und Unruhen auf. $^{44}$ In Müllers Collagen ergibt sich der unruhige, ,hüpfende“ Rhythmus aus dem Widerspiel von textuellen und lautlichen Ordnungsmustern wie dem Reim und bildhaft-visuelle Redundanzen. Die rhythmisch fortschreitende Textbewegung, in der die ,Angst zum Laufen gebracht ${ }^{`}$ werden soll, gerät immer wieder aus dem Tritt und gefriert zum sinnenthobenen, absurden Schreckensbild. Aus dieser stockenden, sich verhaspelnden und in befremdlichen Tableaus verkapselnden Rhythmik der Collagen resultiert ihre spezifische Atmosphäre, die genau die Waage hält zwischen spielerischer Leichtigkeit und lähmender Beklemmung. Die intermodale Kategorie des

39 „Das Interesse innovatorischer ästhetischer Bewegungen am Rhythmischen ist insbesondere motiviert durch das an Überschreitungen traditioneller Grenzen der Gattungen und Kunstformen" (Ebd., S. 251).

${ }^{40}$ Ebd., S. 254.

${ }^{41}$ Christiane Vogel: La configuration rhythme-attente-surprise dans les Cahiers de Paul Valéry, in: Colloquium Helveticum 2002, 32, S. 163-178, hier S. 178.

${ }^{42}$ Vgl. Gerhard Kurz: Macharten. Über Rhythmus, Reim, Stil und Vieldeutigkeit, Göttingen 1999 , S. 13.

${ }^{43}$ Gesetz des Rhythmus ist zugleich Norm und Abweichung, er zeichnet sich durch ,regelmäßige Unregelmäßigkeit“ aus (Helmuth Plessner, zit. nach Waldenfels: Sinnesschwellen, S. 65).

${ }^{44}$ Vgl. Lommel: Der Rhythmus als intermodale Kategorie, S. 81. 
Rhythmus erlaubt es damit, die Collagen in ihrer vibrierenden Gleichzeitigkeit von Stillstand und Bewegung, Bild und Text, mithin in ihrer medienüberschreitenden Transgressivität in den Blick zu nehmen.

\section{Rhythmus als Problemkonstante der Übersetzung intermedialer Gebilde}

Die Kategorie des Rhythmus stellt zugleich ein Bewertungskriterium für die Übersetzung dar. Im Folgenden soll anhand einzelner Leistungen von Leszek Szaruga und Andrzej Kopacki Rhythmus als Problemkonstante in der Übersetzung von Müllers Collagen herausgestellt werden. Das erste Beispiel stammt aus dem Band Vater telefoniert mit den Fliegen:

\footnotetext{
Ach, ein so leichtes

Fleisch hat der Verrat

Rennt und rennt wild und Pfauenblau

Wie die Spiegelenden dieses kurzen

Sommers der das Dorf verbrennt
}

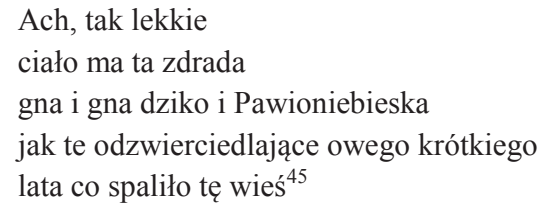

In der Collage wird ein Widerspiel zwischen den visuellen und textuellen Strukturierungen inszeniert. Durch die Anordnung der durch Buchstabengröße und Faktur den Text dominierenden Elemente „Fleisch“, „Verrat“ und „Dorf“, die sich zu einer Phantasmagorie des Entsetzens vernetzen, wird eine simultan-ganzheitliche Wahrnehmung herausgefordert. Die Atmosphäre latenter Bedrohlichkeit ergibt sich zudem aus der medialen Überschreitung zum Bildelement hin, der dunklen Silhouette eines vom Betrachter abgewandten Mannes, wie sie zum Bilderarsenal der Collagen gehören. Der Textteil wird über vage assoziative Zusammenhänge mit der zerschnittenen Birne im Bildteil verzahnt. Der Schnitt durch das Fruchtfleisch der Birne verbindet das Bildelement mit dem im Text erscheinende „Fleisch“, indem er die Vernichtung des unbekümmert-sommerlichen Dorflebens durch den in der Luft liegenden Verrat performativ inszeniert. Bedeutung konstituiert sich über die exponierten Brüche und Schnitte, in denen sich die Verwundungen der Vergangenheit manifestieren. Gegenläufig zur bildhaften Wahrnehmung ist die durch den Text provozierte Lesebewegung. Über die visuelle Strukturierung wird die lautliche geblendet, die über die Redundanz des Elements „rennt“ eine Fluchtbewegung zugleich benennt und sinnfällig macht. Über das Reimen der Wörter „rennt“ und „verbrennt" wird ihre Vergeblichkeit akzentuiert und die Assoziation zu Tod und Vernichtung verstärkt. Die visuellen und lautlichen Strukturierungen vereinigen sich zum spezifischen Rhythmus der Collage und ergänzen sich zu einer Szenerie des

\footnotetext{
${ }^{45}$ Müller: Kolaże, übers. von Leszek Szaruga, S. 90.
} 
lähmenden Schreckens, mit der der Impuls einer Fluchtbewegung alterniert und die Collage zwischen Text und Bild, Erstarrung und Bewegung oszillieren lässt. Das dichte Netz der visuellen, lautlichen und semantischen Bezüge verleiht Müllers Collage eine Kohärenz, die allerdings nicht auf der logischen, sondern affektiven Ebene liegt und im Bereich der Bilder und Vorstellungen operiert. Der Text führt vor, wie Müller die Wort- und Bildelemente nicht nur aus Sinnzusammenhängen herauskatapultiert, sondern sie auch zu neuen zusammenfinden lässt. Das poetische Potenzial der Wörter wird entfaltet und dabei ein stimmiges Vorstellungsbild erzeugt. Für das in den Textteil hineinpurzelnde Wort „pfauenblau“ lässt sich zwar kein semantisch sinnvoller Bezug zu den übrigen Wortelementen etablieren, so dass es als sinnfremdes Element aus dem Text herausragt und den Eindruck des Disparaten erweckt. Über die Farben wird jedoch ein Bezug zwischen dem Wort „Pfauenblau“ und Element „Spiegelenden“ hergestellt, das vor einem pfauenblauen Hintergrund erscheint, womit der Vergleich „Pfauenblau wie die Spiegelenden“ über die visuelle Strukturierung mitvollzogen wird. Das Widerspiel der visuellen und semantischen Strukturierung erzeugt Irritation, durch die sich überlagernden semantischen, visuellen und lautlichen Strukturierungen wird an jeder Stelle Sinn destruiert und neu erzeugt. Auf der Ebene der Assoziationen und Bildvorstellungen entsteht trotz der logischsemantischen Sinnzertrümmerung ein eindrückliches Ganzes mit starker affektiver Wirkung. Es wird hier wie in allen Collagen deutlich, dass Müller, wenn sie neue Wörter zusammensucht, dies tut, um eine Sprache der Opfer zu erfinden. ${ }^{46}$

Dieses Widerspiel von Sinnentfremdung und spielerischer Kontingenz auf der einen Seite und dem Etablieren von kohärenten Vorstellungsbildern auf der anderen Seite, das bei Müller durch die übereinander geblendeten semantischen, visuellen und lautlichen Strukturierungen zustande gebracht wird, bleibt in Szarugas Übersetzung nicht erhalten. Das Moment der Verfremdung überwiegt. Während im Ausgangstext der Textteil durch die grammatischen Bezüge strukturiert ist, die insgesamt gesehen, einen grammatisch korrekten Satz ergeben, der eine "Geschichte“ erzählt, fehlen in der Übersetzung die grammatischen Bezüge. Im Ausgangstext sind die „Spiegelenden“ durch den grammatischen Genitivbezug mit dem Sommer verbunden. Szaruga hingegen scheint das Substantiv „Spiegelenden“ als ein adverbiales „spiegelndes“ (odzwierciedlające) zu lesen, zu dem der Bezug fehlt. Hier wird das Moment des Disparaten verabsolutiert, die fehlende grammatische und semantische Kohärenz wird nicht durch rhythmisch-strukturierende Gestaltungspraktiken wie visuelle oder lautliche Korrespondenzen aufgewogen. Szaruga lässt die Konstellativität der Collagen außer Acht, die von der Linearität wegführt und zwischen einzel-

46 „So haben die Opfer von Grausamkeit, Menschen, die leiden, nicht viel Sprache. Deshalb gibt es so etwas wie „die Stimme der Unterdrückten“ oder ,die Sprache der Opfer“ nicht. Die Sprache, die die Opfer vorher benutzten, passt nicht mehr, und sie leiden zuviel, um neue Wörter zusammensuchen zu können." (Rorty: Kontingenz, Ironie und Solidarität, S. 160). 
nen Elementen Querbezüge zustande bringt, die sich zu irisierender Bildlichkeit verdichten.

So dürfte aufgrund der sich in der Vertikale ergebenden Isotopien (Verrat - Zerstörung - verbrennendes Fleisch) das Element „Fleisch“ sicher nicht als „ciało“ wiedergegeben werden, denn während „,ciało“ auf die organische Ganzheit des menschlichen Körpers verweist, bezeichnet das „Fleisch“ seine schiere Materialität. Szarugas Wiedergabe als „ciało“ zerstört ebenfalls den Bezug Fleisch - Fruchtfleisch, der sich aus den intermedialen Grenzüberschreitungen ergibt, die Bedeutung fluide machen und Signifikanten durch Text- und Bildteile wandern lassen. Es ist zweifelhaft, ob der Übersetzer die Collagen als intermediale Gebilde erkennt, in denen die Visualität mit der semantischen Bedeutung interferiert. Beispielhaft ist das Element „Verrat“, das, mit dem bestimmten Artikel versehen, den Verrat als Phänomen bezeichnet, als besonders schwerwiegenden Vertrauensbruch, der das Gefühl der Selbstverständlichkeit untergräbt und durch ein latentes Gefühl der Angst ersetzt. Diese umfassende, geradezu existenzielle Bedeutung des Verrats wird durch die typografische Gestaltung unterstützt, die das Wortelement die gesamte Collage dominieren lässt. In der Übersetzung von Szaruga wird aus dem bedrohlichen Phänomen des Verrats „ta zdrada“, also ein Einzelgeschehen. Es wurde damit die Intermedialität der Collage nicht erkannt und der Rhythmus, der sich aus der Überlagerung visueller und prosodisch-lautlicher Strukturierungen ergibt, außer Acht gelassen. Während hier die Erstarrung zu einer Szenerie des Schreckens, die sich aus der Verräumlichung des Textes hin zu visuell wahrnehmbaren Konstellationen sowie dem Interferieren von Wort- und Bildelementen ergibt, nicht mitvollzogen wird, so wird an anderer Stelle die Textbewegung missinterpretiert. Der Fluchtimpuls des Weglaufens, der mit dem redundanten Element „rennt“ benannt wird und sich in der durch Metrum und Reim dynamisierten Textbewegung lautlich manifestiert, wird durch ,gnać" wiedergegeben, das ein Hinter-etwas-Herjagen bezeichnet.

Das nächste Beispiel stammt aus dem Bad Die blassen Herren mit den Mokkatassen und beschreibt eine traumatische Situation aus der Ceauşescu-Diktatur, die bereits im Titel des Bandes anklingt: die Verhöre durch die Securitate, bei denen man hilflos gelähmt vor dem Geheimdienstler sitzt.

In einer Knorpeltasse

bot er mir

Einen Kaffee an und der

\section{War schwarzes Haar}

Der Zuckerwürfel weißer Zahn

Na klar fing ich zu rühren an

er sagte Rühr nicht falsch herum

du wirfst den ganzen Sommer um
W filiżance $\mathrm{z}$ chrząstki

ZAproponował mi

KAWE a była ona

Czarna włos

Kostki cukru biały ząb

No jasne 'zaczęłam' mieszać

On powiedział nie MIESZAJ fałszywie

ty to całe lato wywrócisz ${ }^{47}$

\footnotetext{
${ }^{47}$ Müller: Kolaże, übers. von Leszek Szaruga, S. 39.
} 
Dieses unbewegliche Sitzen müssen vor dem Verhörenden mit seiner feisten Behäbigkeit und schmierigen Gebaren wird in der Collage inszeniert. Müller stellt hier in beispielhafter Weise die surreale Welt der Angst dar, die jedes Detail bedrohlich anwachsen lässt und die Welt in sinnlose, bedrohliche Einzelteile zerfallen lässt, in der der Kaffee zu schwarzem Haar und der Zuckerwürfel zum weißen Zahn mutieren. Diese fragmentierten und surreal zusammengeschnittenen Ansichten der Realität werden zugleich zu bizarren Todesvisionen, indem sie das Haar und die Zähne als die leiblichen Überreste eines Menschen exponieren. Die Verknüpfung des Elemente „schwarzes“ und „Haar“ legen zudem einen intertextuellen Verweis auf Celans Todesfuge nahe. Gegen diese Erstarrung zum Schreckensbild, das den Stillstand der Zeit konnotiert, wird im zweiten Teil des Textes eine rhythmisierte Bewegung aufgeboten. Auf semantischer Ebene gibt das „Rühren“ in der Kaffeetasse den Rhythmus der Textbewegung vor, der durch die prosodisch lautliche Strukturierung mit vollzogen wird: Die letzten drei Zeilen sind metrisch geordnet, wobei der Trochäus dominiert, zugleich wird die rhythmische Bewegungsstruktur durch den Reim unterstützt. Durch die ostentative prosodische Strukturierung mutet der Text wie ein Kinderreim an, eine Beschwörungsformel gegen die Angst, mit der Kinder sich zu beruhigen suchen. Damit macht sich der Text zum Schauplatz einer Angstbewältigung, die als zentraler Impuls der Collagen ausgemacht wurde. Der Reim und die Sinnlosigkeit der semantischen Bezüge (den Sommer umwerfen) lassen ein spielerisches Moment anklingen, das dem Text eine grazile Leichtigkeit verleiht.

Der kindliche Ton bleibt jedoch gebrochen. Der unruhige Rhythmus resultiert aus dem Widerspiel von Reim, Metrum und ihrer Überlagerung durch bildhaftvisuelle Strukturen, die die Textbewegung immer wieder ins Stolpern geraten und zu bedrohlichen Szenarien gefrieren lässt. In beispielhafter Weise wird hier ein Spannungsverhältnis zwischen einem spielerischen Umsortieren des vorgefundenen Materials und dessen Verkapselung zu beklemmenden Erinnerungsbildern erzeugt: Zum einen wird das Wortmaterial zu befreiendem Nonsens umgeordnet, wobei die Kontingenz des Sprachspiels durch die heterogene Faktur und Typographie sinnfällig gemacht wird, im Gegenspiel treten assoziative Zusammenhänge hervor, die ein stimmiges Bild der Angst ergeben. Lautliche Redundanzen (,war schwarzes Haar") verleihen dem Text einen düsteren Grundton, der durch die gedämpften Farben verstärkt wird. Es ergibt sich ein beunruhigendes Vorstellungsbild mit starker affektiver Ladung. Die latente Bedrohlichkeit wird durch die mediale Verschränkung mit dem Bildelement, einer dunklen, sich über den Text schiebenden Wolke unterstützt. Der Text oszilliert damit zwischen Bild und Text, Stillstand und Bewegung. Zum einen induzieren die textuellen Strukturierungen wie Syntax, Reim und Metrum eine Lesebewegung, zum anderen bietet er sich ganzheitlicher Betrachtung dar, in der die Konfiguration der Elemente „Haar" und „Zahn“ simultan wahrgenommen wird. Die Collage inszeniert damit beispielhaft das Widerspiel von Schreckensbild und dem Versuch, sich von der Angst freizuschreiben. 
Die Lauttextur, von der Müllers Collage durchzogen ist, geht in der Übersetzung völlig verloren. Es fehlen Reime, Metrum und die Redundanzen der dunklen Vokale. Die Brüchigkeit, die aus dem spielerischen Ton des Kehrreims und der düsteren Bildlichkeit resultiert, fehlt, der gebrochene Rhythmus, der zwischen Stillstand und Bewegung, Bild und Text oszilliert, wird nicht wiedergegeben. Die Kontingenz des Sprachspiels und die Brüchigkeit der Bezüge, die in Müllers Collage durch die Materialität und Medialität der Elemente, ihre heterogene Farblichkeit, Faktur und Buchstabengestaltung und die Schnitte zustande kommt, die teilweise mitten durchs Wort gehen, wird durch die variierte Typographie angedeutet. Die semantische und syntaktische Heterogenität wird in der Übersetzung verstärkt. Während bei Müller das Attribut „schwarzes“ zwar assoziativ mit dem Kaffee verbunden ist, grammatisch aber dem „Haar“ zugeschlagen wird, wird im Translat dieser Zusammenhang zerstört, nur der Kaffee ist schwarz. Da zudem nicht verstanden wurde, dass „Haar“ von Müller hier als plurale tantum verwendet wird und im Translat als Einzelhaar erscheint, hat dies einen gravierenden Eingriff in die Vorstellungswelt des Textes zur Folge.

Ähnlich verhält es sich mit dem „Zuckerwürfel“, der bei Müller zu einem weiBen Zahn mutiert und ein beklemmend-eindringliche Vorstellungsbild erzeugt, dass bei Szaruga durch den im Plural erscheinenden Zuckerwürfel zerstört wird, womit das transgressive Vermögen der einzelnen Bilder abgebremst wird. Es zeigt sich auch hier, dass Szaruga der Spannung zwischen der spielerischen Kontingenz von Müllers Sprachspielen, und dem Etablieren äußerst stimmiger, bedrohlicher Bilder nicht gelungen ist. Er ignoriert die synästhetischen Verfahren Müllers, erkennt die Collagen nicht als visuelle Lautgestalten, die beständig die Schwelle von Text und Bild umspielen. Die spielerisch-befreiende Kontingenz, die sich in steter Spannung gegenüber affektiv-eindrücklichen Vorstellungs- und Erinnerungsbilder behaupten muss, verläuft sich im Translat zu schierer Sinnlosigkeit. Zwar erkennt Szaruga die syntaktische Mehrbezüglichkeit und die semantische Mehrdeutigkeit der Elemente, es gelingt ihm jedoch nicht, sie zu stimmigen Vorstellungsbildern zu verbinden.

Das folgende Translat von Kopacki stammt aus Die blassen Herren mit den Mokkatassen:

Der Schlaf warf mit der schwarzen Schnur

Dran lief das Nachthalshuhn mit dem Gesicht

des Briefträgers und Mutters Hochzeitbildfrisur

Als ich vom Sackbahnhof vier Uhr

Durchs Maisfeld immer nur durchs s e 1 b e

haushoch gelbe Maisfeld zum Vater ins

Gefängnis fuhr
Sen rzucił czarny sznurek wiódł na nim nockę gołoszyjkę miała twarz listonosza i fryzurę matki z monidła kiedy o czwartej za stacji czołowej kukurydzowiskiem wciąż tym samym żółto drapiącym chmurę kukurydzowiskiem jechałam do ojca który siedział za murem ${ }^{48}$

\footnotetext{
${ }^{48}$ Müller: Kolaże, übers. von Andrzej Kopacki, S. 47.
} 
Der Text ist stark rhythmisiert. Grundmetrum ist der Trochäus, der nur zu Beginn der vorletzten Zeile in den Jambus überwechselt. Die durch Reim (Reimschema: $\mathrm{A}-\mathrm{B}-\mathrm{A}-\mathrm{A}-\mathrm{C}-\mathrm{D}-\mathrm{A}$ ) und Metrum erzielte Dynamik der Textbewegung korrespondiert mit dem atemlosen Laufen durch das Maisfeld, wobei die semantischen Bezüge durch die typographische Gestaltung unterstützt werden, indem das Wort „lief“ im Kursivdruck erscheint und so die Bewegung grafisch nachbildet. Der Reim wird hier wiederum als Bewältigungsstrategie wirksam, als Versuch, gegen die Angst anzuschreiben. Der spielerische Umgang mit dem Sprachmaterial, der es zu befreiendem Nonsens umordnet, ist als ästhetische Fluchtbewegung vor schmerzhaften Erinnerungen zu lesen. Indem im Sprachspiel Bedeutung fluide gemacht wird, sollen traumatische Verhärtungen aufgebrochen werden. Die Elemente finden aber immer wieder zusammen zum bedrückenden Erinnerungsbild und machen das vorgefundene Material durchlässig für die dem Kind zugefügten Verwundungen. Die Dynamik der Textbewegung wird immer wieder unterbrochen, die lautlichprosodische Rhythmisierung wird durch ein intrigierendes Wechselspiel mit der visuellen Strukturierung durchkreuzt, indem die Elemente graphisch hervorgehoben werden, die auf die Senkungen des Metrums fallen. Dadurch wirkt der Rhythmus gebrochen, und es wird die „hüpfende Zerbrochenheit“ erzielt, die immer wieder am Horizont von Müllers Collagen-Poetik aufscheint.

Die ästhetische Fluchtbewegung, in der sich die Autorin vom Phantasma der Vergangenheit freischreiben will, wird durch die semantischen und lautlichen Redundanzen („durchs Maisfeld immer nur durchs selbe haushoch gelbe Maisfeld“) abgebremst, die die Insistenz des einschießenden Erinnerungsbildes sinnfällig machen. Die rumänischen Maisfelder, die in ihrer Endlosigkeit die Hoffnungslosigkeit einer zum Stillstand gerinnenden Zeit der Diktatur versinnbildlichen, beschreibt Müller in Mein Vaterland war ein Apfelkern. ${ }^{49}$

Die lautliche Rhythmisierung interferiert auf der visuellen Ebene mit der Redundanz der Farben, die eine weitere rhythmisch wirksame Wiederholungsstruktur darstellt. Das Wort „ich“ erscheint auf grünem Hintergrund, der sich im Bildteil wiederholt, während die mit dem Vater und dem Gefängnis verbundenen Elemente in Schwarz-Weiß-Kontrasten gehalten sind. Die Collage ist damit über die medialen Grenzen hinweg lautlich und visuell rhythmisiert. $\mathrm{Zu}$ den horizontalen, sich mit der Textbewegung entfaltenden Bezügen kommen Korrespondenzen, die sich quer zum Text ergeben und Vertikalen aufreißen. Von dem Wortelement „Gefängnis“, das aufgrund seiner Größe und des schwarzen Fettdrucks den Textteil dominiert, verläuft eine assoziative Achse zu dem in den Bildteil über dem Text montierten Arm, der mit seinem schwarzweiß gestreiften Ärmel an eine Gefängniskluft erinnert. Durch die sich überlagernden textuellen und visuellen Strukturierungen wird ein beständiges Umschwenken vom Modus des Betrachtens zur Lesebewegung initiiert,

\footnotetext{
${ }^{49}$ Müller: Mein Vaterland war ein Apfelkern, S. 7.
} 
womit die Collage sich einer intermedialen Synthese verweigert. Durch die Bezüge zwischen Text und Bild wird vielmehr der Transfer zwischen den Medien permanent aktiv gehalten und jenseits fixer Entitäten eine mediale Hybridität erzeugt, die neue Verweisstrukturen generiert. So verweisen die harten Schnitte im Bildteil der Collage auf die Pein und Gewaltsamkeit der Trennung, den Riss, der mitten durch die Welt und den Menschen geht.

Kopacki hat augenscheinlich die Bedeutung des Rhythmus erkannt. Der Reim wird, wenn auch zuweilen an anderer Stelle als Binnenreim beibehalten (sznurek fryzurę - chmurę - żółto - który - murem), was stark rhythmisierende Redundanzen erzeugt, der derjenigen des Ausgangstextes nicht nachsteht. Der Übersetzer ist bei der Suche und Integrierung von Reimwörtern äußerst kreativ, wenn er das Wort „Gefängnis“ durch „za murem“ ersetzt, um den Reim zu erhalten. Der sorgfältig herausgearbeitete Reim übernimmt die prosodische Funktion des Metrums. Die Dynamik der Textbewegung, die sich von dem traumatischen Erlebnis freischreiben will, bleibt erhalten.

Die spielerische Kontingenz des Sprachspiels, die in Müllers Collage über die Materialität der ausgeschnittenen und neu zusammengefügten Wortelemente erzielt wird, wird im Translat über den Neologismus „kukurydzowisko“ akzentuiert, der zugleich auf die 'Privatsprache" (Wittgenstein) zwischen Vater und Kind und damit auf emotionale Verbundenheit verweist. Die konsequent durchgehaltenen enjambements öffnen den Text auf semantische Mehrbezüglichkeit hin und realisieren einen spielerischen Umgang mit dem Sprachmaterial, der der Poetik von Müllers Collage entspricht. Auf der anderen Seite wird die Insistenz der Erinnerung an den Verlusts des Vaters, die in der Collage über die Bildlichkeit und Konstellativität der Bildund Wortelemente sinnfällig gemacht wird, durch die semantischen Redundanzen (,kukurydzowiskiem wciąż tym samym żółto drapiącym chmurę kukurydzowiskiem") beibehalten, die gleichermaßen den Grundaffekt der Angst und Verzweiflung vermitteln. Die Spannung zwischen spielerisch dynamischer Textbewegung und der Eindringlichkeit des Erinnerungsbildes, die den Rhythmus der Collage ausmacht, wird damit von Kopacki wiedergegeben.

Der Übersetzer von Müllers Collagen bewegt sich innerhalb einer vielschichtigen Konstellation von Medien. Die Übersetzung kann nur dann glücken, wenn erkannt wird, dass Bedeutungskonstitution sich über das intermediale Widerspiel von textuellen, semantischen, lautlich-prosodischen und visuellen Bezügen vollzieht. Im Zusammenspiel ergeben sie den Rhythmus der Collagen, der über das kognitive Verstehen hinaus subkutan wirksam wird und eine affektive Aufladung der Collagen bewirkt. Seine Gebrochenheit reflektiert das unaufhebbare Spannungsverhältnis zwischen den ästhetischen Fluchtbewegungen der in den Collagen inszenierten spielerischen Kontingenz, die in bester avantgardistischer Tradition zum Nonsens gerät, und den Schreckensbildern, zu denen sich das Sprachspiel der Collagen immer wieder verdichtet. 


\section{Literatur}

Deleuze, Gilles: Francis Bacon - Logik der Sensation. Aus dem Franz. von Joseph Vogl, München 1995.

Eke, Norbert Otto: Schönheit der Verwund(er)ung. Herta Müllers Weg zum Gedicht, in: Herta Müller. Text + Kritik, hrsg. von Heinz-Ludwig Arnoldt, München 2002, S. 64-79.

Faust, Wolfgang Max: Bilder werden Worte. Zum Verhältnis von bildender Kunst und Literatur vom Kubismus bis zur Gegenwart. Köln 1987.

Kurz, Gerhard: Macharten. Über Rhythmus, Reim, Stil und Vieldeutigkeit, Göttingen 1999.

Lommel, Michael: Der Rhythmus als intermodale Kategorie, in: Intermedialität analog/ digital. Theorien - Methoden - Analysen, hrsg. von Joachim Paech und Jens Schröter, München 2008, S. 79-90.

Lommel, Michael: Samuel Beckett - Synästhesie als Medienspiel, München 2006.

Lommel, Michael: Stimme und Blick. Paradoxien synästhetischer Medienrezeption, in: Navigationen 2002, Jg. 1, Nr. 2, S. 7-16.

Merleau-Ponty, Maurice: Phénoménologie de la Perception, Paris 1966.

Müller, Herta: Der fremde Blick oder Das Leben ist ein Furz in der Laterne, Göttingen 1999.

Müller, Herta: Der Teufel sitzt im Spiegel. Wie Wahrnehmung sich erfindet, Berlin 1991.

Müller, Herta: Der Wächter nimmt seinen Kamm, Reinbek bei Hamburg 1993.

Müller, Herta: Die blassen Herren mit den Mokkatassen, München 2005.

Müller, Herta: Hunger und Seide, Reinbek bei Hamburg 1995.

Müller, Herta: Im Haarknoten wohnt eine Dame, Reinbek bei Hamburg 2000.

Müller, Herta: In der Falle, Göttingen 1996.

Müller, Herta: Ich habe die Sprache gegessen. Die Literatur-Nobelpreisträgerin Herta Müller über ihren zusammengeklebten Gedichte und über die Macht und das Versagen der Wörter, in: Der Spiegel 2012, 35, S. 128-132.

Müller, Herta: Kolaże, übers. von Leszek Szaruga, Wrocław 2013.

Müller, Herta: Kolaże, übers. von Andrzej Kopacki, in: Literatura na Świecie 2014, Nr. 1-2, S. 133-156.

Müller, Herta: Mein Vaterland war ein Apfelkern. Ein Gespräch mit Angelika Klammer, München 2014.

Müller, Herta: Mit dem Auge kann man keinen Stift halten, S. 1-3, <http://derstandard.at/1537469>, (Zugriff am 03.07.2014).

Müller, Herta: Vater telefoniert mit den Fliegen, Frankfurt a.M. 2012.

Müller, Herta, Eddy, Beverly Driver: Die Schule der Angst. Gespräch mit Herta Müller vom 14. April 1998, in: The German Quaterly 1999, 72.4, S. 329-339.

Müller, Herta, Kopacki, Andrzej: Szczegót nie kłamie, z Herta Müller rozmawia Andrzej Kopacki, in: Literatura na Świecie 2014, Nr. 1-2, S. 157-163.

Müller, Philipp: Fluchtlinien der erfundenen Wahrnehmung. Strategien der Überwachung und minoritäre Schreibformen in Herta Müllers Roman „Heute wäre ich mir lieber nicht begegnet, in: Herta Müller. Text + Kritik 155, hrsg. von Heinz-Ludwig Arnold, München 2002, S. 49-58.

Rorty, Richard: Kontingenz, Ironie und Solidarität. Aus dem Amerikanischen von Christa Krüger, Frankfurt a.M. 1989.

Schmitz-Emans, Monika: Rhythmisierung als Musikalisierung. Zu Selbstbeschreibungen und ästhetischer Praxis in der experimentellen Dichtung des 20. Jahrhunderts, in: Colloquium Helveticum 2002, 32, S. 243-887.

Tzara, Tristan: Dada Manifest über die aschwache Liebe und die bittere Liebe, in: Ders.: 7 DADA Manifeste, Hamburg 1978.

Vogel, Christiane: La configuration rhythme-attente-surprise dans les Cahiers de Paul Valéry, in: Colloquium Helveticum 2002, 32, S. 163-178. 
Waldenfels, Bernhard: Sinnesschwellen: Studien zur Phänomenologie des Fremden 3, Frankfurt a.M. 1999.

Wertheimer, Jürgen: Im Papierhaus wohnt die Stellungnahme. Zu Herta Müllers Bild-Text-Collagen, in: Herta Müller. Text + Kritik 155, hrsg. von Heinz-Ludwig Arnold, München 2002, S. 80-84. 